\title{
POSITIVE EIGENVALUES AND TWO-LETTER GENERALIZED WORDS*
}

\author{
C. HILLAR ${ }^{\dagger}$, C. R. JOHNSON ${ }^{\ddagger}$, AND I. M. SPITKOVSKY $\ddagger$
}

\begin{abstract}
A generalized word in two letters $A$ and $B$ is an expression of the form $W=$ $A^{\alpha_{1}} B^{\beta_{1}} A^{\alpha_{2}} B^{\beta_{2}} \cdots A^{\alpha_{N}} B^{\beta_{N}}$ in which the exponents are nonzero real numbers. When independent positive definite matrices are substituted for $A$ and $B$, it is of interest whether $W$ necessarily has positive eigenvalues. This is known to be the case when $N=1$ and has been studied in case all exponents are positive by two of the authors. When the exponent signs are mixed, however, the situation is quite different (even for 2 -by-2 matrices), and this is the focus of the present work.
\end{abstract}

Key words. Positive definite matrices, projections, generalized word

AMS subject classifications. 15A18, 15A57

Let $A, B$ be positive definite $n \times n$ matrices. Then, as is well known [5, p. 465], the eigenvalues of the product $A B$ are real and positive. Moreover, for all $\alpha, \beta \in \mathbb{R}$ the matrices $A^{\alpha}$ and $B^{\beta}$ are positive definite together with $A, B$. Thus, the eigenvalues of $A^{\alpha} B^{\beta}$ are real and positive as well.

In this paper, we are concerned with possible generalizations of this simple observation to products $W(A, B)=A^{\alpha_{1}} B^{\beta_{1}} A^{\alpha_{2}} \ldots$. Such expressions, when the $\alpha$ 's and $\beta$ 's are positive integers, have been studied in [4] and when $\alpha$ 's and $\beta$ 's are positive reals in subsequent work. Applying an appropriate similarity if necessary, we may without loss of generality suppose that $W(A, B)$ ends with a power of $B$. In other words,

$$
W(A, B)=A^{\alpha_{1}} B^{\beta_{1}} A^{\alpha_{2}} B^{\beta_{2}} \cdots A^{\alpha_{N}} B^{\beta_{N}} \quad\left(\alpha_{j}, \beta_{j} \in \mathbb{R} \backslash\{0\}\right) .
$$

We will say that (1) is a generalized word (g-word) in $A, B$ of class $N$.

Problem. Under what additional conditions on $A, B$ and/or the structure of the $g$-word (1) is it true that all the eigenvalues of $W(A, B)$ are positive?

The above observation means that there are no additional conditions on $A$ and $B$ for $g$-words of class 1 . Another trivial sufficient condition is the commutativity of $A$ and $B$ (which holds, in particular, for $n=1$ ). Starting with $n=N=2$, it is easy to give examples of g-words (1) with positive definite $A, B$ and the spectrum not lying in $\mathbb{R}_{+}$. The simplest such word is $A B A^{-1} B^{-1}$. That this word does not guarantee positive spectrum can be seen from the following, more precise, statement.

THEOREM 1. Let $A$ have exactly two distinct eigenvalues. Then the spectrum of $A^{m} B A^{-m} B^{-1}$ is positive for all $m \in \mathbb{N}$ if and only if $A$ and $B$ commute.

\footnotetext{
${ }^{*}$ Received by the editors on 12 September 2001. Accepted for publication on 27 January 2002. Handling Editor: Miroslav Fiedler.

${ }^{\dagger}$ Mathematics Department, University of California, Berkeley Berkeley, CA 94720, USA (chillar@uclink.berkeley.edu). Supported under a National Science Foundation Graduate Research Fellowship.

¥Department of Mathematics, College of William \& Mary, Williamsburg, VA 23187-8795, USA (crjohnso@math.wm.edu, ilya@math.wm.edu). This research was supported by NSF REU Grant DMS 99-87803.
} 
Proof. Using a unitary similarity if necessary, we may put $A$ in the form

$$
A=\left[\begin{array}{cc}
\lambda_{1} I_{n_{1}} & 0 \\
0 & \lambda_{2} I_{n_{2}}
\end{array}\right]
$$

where $\lambda_{1}>\lambda_{2}>0$; denote the respective partition of $B$ by

$$
B=\left[\begin{array}{ll}
B_{11} & B_{12} \\
B_{21} & B_{22}
\end{array}\right]
$$

(due to self adjointness of $B$, the blocks $B_{11}, B_{22}$ also are self adjoint, and $B_{21}=B_{12}^{*}$ ). Then

$$
A^{m} B A^{-m}=\gamma^{-m}\left[\begin{array}{cc}
\gamma^{m} B_{11} & B_{12} \\
\gamma^{2 m} B_{21} & \gamma^{m} B_{22}
\end{array}\right]
$$

where $\gamma=\lambda_{2} / \lambda_{1}<1$. Thus, there exists the limit of $\gamma^{m} A^{m} B A^{-m} B^{-1}$ when $m \rightarrow \infty$, and this limit equals

$$
\left[\begin{array}{cc}
-B_{12} C^{-1} B_{21} B_{11}^{-1} & B_{12} C^{-1} \\
0 & 0
\end{array}\right]
$$

where $C=B_{22}-B_{21} B_{11}^{-1} B_{12}$ is positive definite due to the positive definiteness of $B$ (see, e.g., [5, p. 475]). Suppose that the eigenvalues of all the matrices $A^{m} B A^{-m} B^{-1}$ are positive. Then all the eigenvalues of the left upper block of the matrix (2) are non-negative. In other words, the spectrum of the matrix $B_{12} C^{-1} B_{21} B_{11}^{-1}$ must be non-positive. The latter being a product of a non-negative definite matrix $B_{12} C^{-1} B_{21}$ and a positive definite matrix $B_{11}^{-1}$, this is only possible if it is the zero matrix. But then $0=B_{12} C^{-1} B_{21}=\left(B_{12} C^{-1 / 2}\right)\left(B_{12} C^{-1 / 2}\right)^{*}$, so that $B_{12}=0$. This implies that $B_{21}=0$ as well. In other words, $B$ commutes with $A$.

Observe that in Theorem 1 both $A$ and $B$ appear with powers of different sign, and that for the g-words of class 1 this situation is impossible. So, it is natural to entertain a conjecture that g-words (1) with powers of the same sign have positive spectra. As it happens, this is also not true (even for words of class 2 and natural exponents) but the respective example is much harder to come by. The simplest known example of this kind is the word $A B A^{2} B^{2}$, with

$$
A=\left[\begin{array}{ccc}
1 & 20 & 210 \\
20 & 402 & 4240 \\
210 & 4240 & 44903
\end{array}\right], \quad B=\left[\begin{array}{ccc}
36501 & -3820 & 190 \\
-3820 & 401 & -20 \\
190 & -20 & 1
\end{array}\right]
$$

(see [4]). Note that in (3) $n=3$ and all the eigenvalues of both matrices $A$ and $B$ are distinct. The next two theorems show that these features are indeed necessary for such an example. Let us prove an auxiliary statement first.

LEMma 2. Let one of the matrices $A, B$ have an eigenvalue of multiplicity at least $n-1$ and in (1) all the powers of the other matrix be of the same sign. Then $W(A, B)$ has at least one positive eigenvalue. 
Proof. Without loss of generality (by a simple change of notation if necessary) we may suppose that $A$ is the matrix with an eigenvalue $\lambda_{1}$ of multiplicity $n-1$; denote its remaining eigenvalue by $\lambda_{2}$. Switching from $B$ to $B^{-1}$ if necessary, we may also suppose that $\beta_{1}, \ldots, \beta_{N} \geq 0$.

Case 1. $\beta_{1}, \ldots, \beta_{N}$ are integers. Let $U$ be a unitary similarity diagonalizing $A$ :

$$
A_{0}=U^{*} A U=\left[\begin{array}{ccccc}
\lambda_{1} & 0 & \ldots & 0 & 0 \\
0 & \lambda_{1} & & 0 & 0 \\
\vdots & & \ddots & \vdots & \vdots \\
0 & \ldots & 0 & \lambda_{1} & 0 \\
0 & 0 & \ldots & 0 & \lambda_{2}
\end{array}\right]
$$

By an appropriate choice of $U$ (which consists in multiplying the original one on the right by $V \oplus[1]$, where $V$ is some $(n-1) \times(n-1)$ unitary matrix), we may suppose that the left upper $(n-1) \times(n-1)$ block of $B$ also is diagonalized. Multiplying $V$ on the right by a diagonal unitary matrix with suitably chosen arguments of its diagonal entries, we can force all the elements of the last column in $B_{0}=U^{*} B U$ to become non-negative. But then all elements of its last row automatically become nonnegative as well. In other words, simultaneously with (4) the following decomposition also holds:

$$
B_{0}=U^{*} B U=\left[\begin{array}{ccccc}
\mu_{1} & 0 & \ldots & 0 & \gamma_{1} \\
0 & \mu_{2} & & 0 & \gamma_{2} \\
\vdots & & \ddots & \vdots & \vdots \\
0 & \ldots & 0 & \mu_{n-1} & \gamma_{n-1} \\
\gamma_{1} & \gamma_{2} & \ldots & \gamma_{n-1} & \mu_{n}
\end{array}\right]
$$

Both matrices $A_{0}$ and $B_{0}$ are (entry-wise) non-negative. Thus, $W\left(A_{0}, B_{0}\right)$ also is entry-wise non-negative, and (at least) one of its eigenvalues is positive due to Perron's theorem. But $W\left(A_{0}, B_{0}\right)=U^{*} W(A, B) U$, and the result follows.

Case 2. $\beta_{1}, \ldots, \beta_{N}$ are rational. Let $Q(\in \mathbb{N})$ be their least common denominator. Considering $B^{1 / Q}$, we reduce this situation to Case 1 .

Case 3. Arbitrary (non-negative) $\beta_{1}, \ldots, \beta_{N}$. For each $j=1, \ldots, N$, introduce a sequence $\beta_{j}^{(k)}$ of non-negative rational numbers such that $\lim _{k \rightarrow \infty} \beta_{j}^{(k)}=\beta_{j}$. Let

$$
W_{k}(A, B)=A^{\alpha_{1}} B^{\beta_{1}^{(k)}} A^{\alpha_{2}} B^{\beta_{2}^{(k)}} \cdots A^{\alpha_{N}} B^{\beta_{N}^{(k)}} .
$$

Then each of the matrices $W_{k}(A, B)$ has a positive eigenvalue (due to Case 2), and their limit $W(A, B)$ is invertible. From continuity considerations it follows that $W(A, B)$ also has a positive eigenvalue.

TheOREM 3. Let $n=2$, and let all powers of either $A$ or $B$ in (1) be of the same sign. Then all the eigenvalues of $W(A, B)$ are positive.

Proof. Since $n-1=1$, both $A$ and $B$ have eigenvalues of multiplicity $n-1$. Hence, conditions of Lemma 2 are satisfied, so that at least one eigenvalue of $W(A, B)$ is positive. But the product of the two eigenvalues, det $W(A, B)$, is positive as well. Thus, the second eigenvalue is also positive. 
THEOREM 4. Let $n=3$, and suppose that at least one of the matrices $A, B$ has a multiple eigenvalue. If all the powers of the other matrix in (1) are of the same sign, then all the eigenvalues of $W(A, B)$ are positive.

Proof. Since $n-1=2$, conditions of Lemma 2 are met. We will use representations (4), (5) from its proof, which in case $n=3$ take the form

$$
A_{0}=\left[\begin{array}{ccc}
\lambda_{1} & 0 & 0 \\
0 & \lambda_{1} & 0 \\
0 & 0 & \lambda_{2}
\end{array}\right], \quad B_{0}=\left[\begin{array}{ccc}
\mu_{1} & 0 & \gamma_{1} \\
0 & \mu_{2} & \gamma_{2} \\
\gamma_{1} & \gamma_{2} & \mu_{3}
\end{array}\right]
$$

If $\gamma_{1}=0$ or $\gamma_{2}=0$ then $A_{0}$ and $B_{0}$ are simultaneously in the block diagonal form, so that $W\left(A_{0}, B_{0}\right)$ is a direct sum of a positive scalar and $W\left(A_{1}, B_{1}\right)$, where $A_{1}$ and $B_{1}$ are $2 \times 2$ positive definite matrices. The result then follows from Theorem 3 .

If both $\gamma_{1}$ and $\gamma_{2}$ are strictly positive, we will again consider first the case of natural powers of $B$. There is no need to consider the case $N=1$; in all other cases $W\left(A_{0}, B_{0}\right)$ is entry-wise positive. According to Perron's theorem, its positive eigenvalue $\eta_{1}$ coinciding with the spectral radius is the only eigenvalue of this magnitude. Thus, $\eta_{1}$ is the eigenvalue of $W(A, B)$ and the other two eigenvalues satisfy $\left|\eta_{3}\right| \leq\left|\eta_{2}\right|<\eta_{1}$. Observe now that $W(A, B)^{-1}$ is a word in $A^{-1}, B^{-1}$, and that $A^{-1}$, $B^{-1}$ satisfy conditions of Lemma 2 simultaneously with $A, B$. Thus, the biggest by its absolute value eigenvalue $\eta_{3}^{-1}$ of $W(A, B)^{-1}$ must be positive as well. From this, and the positivity of $\operatorname{det} W(A, B)=\eta_{1} \eta_{2} \eta_{3}$ we conclude that the remaining eigenvalue $\eta_{2}$ is also positive.

The case of arbitrary real $\beta_{1}, \ldots, \beta_{N}$ of the same sign can be now covered in exactly the same manner as in the proof of Lemma 2 .

Our next result shows that in Theorem 3 it is not the size of the matrices that counts but actually the number of their distinct eigenvalues.

TheOREM 5. Suppose that each of the matrices $A$ and $B$ has at most two distinct eigenvalues and that in (1) all powers of either $A$ or $B$ are of the same sign. Then, for an arbitrary $n$, all the eigenvalues of $W(A, B)$ are positive.

Proof. If $\lambda_{1}$ and $\lambda_{2}$ are the only eigenvalues of $A$, then $A=\left(\lambda_{1}-\lambda_{2}\right) P+\lambda_{2} I$, where $P$ is a certain orthoprojection. Similarly, $B=\left(\mu_{1}-\mu_{2}\right) Q+\mu_{2} I$, where $Q$ is another orthoprojection. It is well known (see, e.g., [1], [2], or [3]) that, for any two orthoprojections $P$ and $Q$, there is a unitary similarity $U$ such that

$$
P_{0}=U^{*} P U=P_{1} \oplus P_{2} \oplus \cdots \oplus P_{N}, Q_{0}=U^{*} Q U=Q_{1} \oplus Q_{2} \oplus \cdots \oplus Q_{N},
$$

where the size of $P_{j}$ is the same as the size of $Q_{j}$ and does not exceed $2(j=1, \ldots, N)$. But then

$$
U^{*} W(A, B) U=W\left(A_{1}, B_{1}\right) \oplus W\left(A_{2}, B_{2}\right) \oplus \cdots \oplus W\left(A_{N}, B_{N}\right),
$$

where $A_{j}=\left(\lambda_{1}-\lambda_{2}\right) P_{j}+\lambda_{2} I, B_{j}=\left(\mu_{1}-\mu_{2}\right) Q_{j}+\mu_{2} I$ are either positive numbers or positive definite $2 \times 2$ matrices. Due to Theorem 3 , the eigenvalues of $W\left(A_{j}, B_{j}\right)$ are all positive. The same is true for their direct sum $U^{*} W(A, B) U$, and thus for $W(A, B)$ itself. 
Let us say that the sequence $\alpha_{1}, \beta_{1}, \ldots, \alpha_{N}, \beta_{N}\left(\in(\mathbb{R} \backslash\{0\})^{2 N}\right)$ is 2-good if the word (1) has positive eigenvalues for all positive definite $2 \times 2$ matrices $A, B$. Of course, $k$-good sequences can be defined in a similar way for any $k \in \mathbb{N}$, and every $k$-good sequence is also $j$-good for $j<k$. According to Theorem 5 , any sequence for which either all $\alpha$ 's or all $\beta$ 's are of the same sign is 2-good. Many such sequences are $k$-good for all positive integers $k$, as discussed in [4]. On the other hand, Theorem 1 implies that the sequence $\alpha, \beta,-\alpha,-\beta$ is not 2 -good. In fact, the magnitudes of the exponents are in this case irrelevant: any sequence $\alpha_{1}, \beta_{1}, \alpha_{2}, \beta_{2}$ with $\alpha_{1} \alpha_{2}<0$, $\beta_{1} \beta_{2}<0$ is not 2 -good. This statement is a particular case of a more general one, the formulation of which requires some preparation.

Consider the following cancellation rule for the sequences $\alpha_{1}, \beta_{1}, \ldots, \alpha_{m}, \beta_{m}, m \in$ $\mathbb{N}$ : if $\alpha_{j} \alpha_{j+1}>0$ for some $j \in\{1, \ldots, m\}$ (where by convention $\alpha_{m+1}=\alpha_{1}$ ), then $\alpha_{j}, \beta_{j}$ are omitted from the sequence. Similarly, if $\beta_{j} \beta_{j+1}>0$ then $\alpha_{j+1}, \beta_{j+1}$ are omitted. The sequence $\alpha_{1}, \beta_{1}, \ldots, \alpha_{m}, \beta_{m}$ is irreducible if no cancellations (in the above sense) are possible. Observe that the signs of both $\alpha_{1}, \alpha_{2}, \ldots$ and $\beta_{1}, \beta_{2}, \ldots$ in an irreducible sequence alternate. We will say that $m$ is the reduced class of the sequence $\alpha_{1}, \beta_{1}, \ldots, \alpha_{N}, \beta_{N}$ if there is an irreducible sequence consisting of $2 m$ terms obtained from $\alpha_{1}, \beta_{1}, \ldots, \alpha_{N}, \beta_{N}$ by a repeated application of the cancellation rule.

TheOrem 6. Any sequence $\alpha_{1}, \beta_{1}, \ldots \alpha_{N}, \beta_{N}$ of the reduced class $m \equiv 2$ or 3 $\bmod 4$ is not 2-good.

Proof. Switching from $A$ to $A^{-1}$ and/or from $B$ to $B^{-1}$ if necessary, we may without loss of generality suppose that the first $\alpha$ and $\beta$ remaining after the cancellation procedure are both positive. Then let

$$
A=\left[\begin{array}{ll}
1 & 0 \\
0 & \epsilon
\end{array}\right], \quad B=\left[\begin{array}{cc}
1 / 2+\epsilon & 1 / 2 \\
1 / 2 & 1 / 2
\end{array}\right]
$$

for some $\epsilon>0$. An easy computation shows that the matrix

$$
2^{\sum_{\beta_{j}<0} \beta_{j}} \epsilon^{-\left(\sum_{\alpha_{j}<0} \alpha_{j}+\sum_{\beta_{j}<0} \beta_{j}\right)} A^{\alpha_{1}} B^{\beta_{1}} A^{\alpha_{2}} B^{\beta_{2}} \cdots A^{\alpha_{N}} B^{\beta_{N}}
$$

is the product of $2 N$ matrices the $(2 j-1)$-st of which is $\left[\begin{array}{cc}1 & 0 \\ 0 & \epsilon^{\alpha_{j}}\end{array}\right]$ if $\alpha_{j}>0$ and $\left[\begin{array}{cc}\epsilon^{-\alpha_{j}} & 0 \\ 0 & 1\end{array}\right]$ if $\alpha_{j}<0$, and the $2 j$-th of which is $\left[\begin{array}{cc}1 / 2+\epsilon & 1 / 2 \\ 1 / 2 & 1 / 2\end{array}\right]^{\beta_{j}}$ if $\beta_{j}>0$ and $\left[\begin{array}{cc}1 / 2 & -1 / 2 \\ -1 / 2 & 1 / 2+\epsilon\end{array}\right]^{-\beta_{j}}$ if $\beta_{j}<0, j=1, \ldots, N$.

Thus, the limit of (7) for $\epsilon \rightarrow 0$ exists and equals

$$
P_{1} Q_{1} P_{2} Q_{2} \cdots P_{N} Q_{N}
$$

where $P_{j}$ is $P=\left[\begin{array}{ll}1 & 0 \\ 0 & 0\end{array}\right]$ if $\alpha_{j}>0$ and $I-P$ if $\alpha_{j}<0$, and $Q_{j}$ is $Q=\left[\begin{array}{cc}1 / 2 & 1 / 2 \\ 1 / 2 & 1 / 2\end{array}\right]$ if $\beta_{j}>0$ and $I-Q$ if $\beta_{j}<0$.

A straightforward computation shows that

$$
P Q P=P(I-Q) P=\frac{1}{2} P,(I-P) Q(I-P)=(I-P)(I-Q)(I-P)=\frac{1}{2}(I-P),
$$


and

$$
Q P Q=Q(I-P) Q=\frac{1}{2} Q,(I-Q) P(I-Q)=(I-Q)(I-P)(I-Q)=\frac{1}{2}(I-Q) .
$$

Consequently (recall the condition imposed on the signs of $\alpha$ 's and $\beta$ 's and the alternating nature of irreducible sequences), the matrix (8), up to a positive scalar multiple $2^{N-m}$, coincides with

$(P Q(I-P)(I-Q))^{m / 2}$ if $m$ is even, and $(P Q(I-P)(I-Q))^{(m-1) / 2} P Q$ if $m$ is odd.

It can be checked by induction that, for any $k \in \mathbb{N}$,

$$
(P Q(I-P)(I-Q))^{k}=\frac{1}{4^{k}}\left[\begin{array}{cc}
(-1)^{k} & (-1)^{k-1} \\
0 & 0
\end{array}\right]
$$

This implies that the trace of (8) in case of odd $\lfloor m / 2\rfloor$ is negative. But then, for sufficiently small $\epsilon>0$, the trace of $A^{\alpha_{1}} B^{\beta_{1}} A^{\alpha_{2}} B^{\beta_{2}} \cdots A^{\alpha_{N}} B^{\beta_{N}}$ also is negative. It remains to observe that $\lfloor m / 2\rfloor$ is odd if and only if $m \equiv 2$ or $3 \bmod 4$.

Theorems 5 and 6 combined give a complete description of all 2-good sequences of class 2. Observe that in this case "2-goodness" does not depend on the magnitude of the elements of the sequence but only on the sign pattern. At the moment, we do not know whether this is true for sequences of arbitrary length. We observe also that representation (6) shows that, if the sequence $\alpha_{1}, \ldots, \beta_{N}$ in (1) is 2 -good, then $W(A, B)$ has positive eigenvalues for matrices $A, B$ of any size, provided that each of them has at most two distinct eigenvalues.

\section{REFERENCES}

[1] C. Davis. Separation of two linear subspaces. Acta Sci. Math. (Szeged), 19:172-187, 1958.

[2] J. Dixmier. Position relative de deux variétés linéaires fermées dans un espace de Hilbert. Revue Scientifique, 86:387-399, 1948.

[3] P. L. Halmos. Two subspaces. Trans. Amer. Math. Soc., 144:381-389, 1969.

[4] C. Hillar and C. R. Johnson. Eigenvalues of words in two positive definite letters. SIAM J. Matrix Anal. Appl., to appear.

[5] R. A. Horn and C. R. Johnson. Matrix Analysis. Cambridge University Press, New York, 1985. 